\title{
Molecular Dynamics and Local Molecular Conformation in Solid Materials Studied by Nuclear Magnetic Resonance
}

\author{
Eduardo Ribeiro deAzevedo and Tito José Bonagamba \\ Instituto de Física de São Carlos, Universidade de São Paulo, \\ Caixa Postal 369, CEP: 13560-970, São Carlos, SP, Brazil
}

Received on 1st October, 2005; accepted on 28 November, 2005

\begin{abstract}
After the huge development occurred during the last 30 years, including the proposition of methods such as magic-angle spinning, multiple-resonance experiments (cross-polarization, high-power decoupling, etc.), multiple-pulses techniques, multiple quantum experiments, and multidimensional spectroscopy, pulsed SolidState NMR became one of the most important experimental tools for studying physical and chemical properties of new materials in the solid-state. In this article we will review some of the NMR methods employed for studying an important class of solid materials: organic materials, particularly polymers and derivatives, which allow the application of a large number of different techniques for understanding their molecular dynamics and local conformation.
\end{abstract}

Keywords: Molecular dynamics; NMR; Organic materials

\section{INTRODUCTION}

Molecular Dynamics and local conformation have important effects on the properties of polymers and derivatives, proteins, pharmaceuticals, amorphous materials near the glass transition, ion transport in organic and inorganic ionic conductors, and other structural properties of organic and inorganic systems. Solid-state NMR provides powerful techniques for elucidating details of segmental dynamics and local conformation in solid materials. NMR methods allow the study of dynamics occurring in a wide frequency range (1 $\mathrm{Hz}$ to $\sim 100 \mathrm{MHz})$. Besides, NMR also provides a series of methods capable of obtaining reliable measurements of torsion angles between localized sites, short and intermediate range structure, as well as interatomic distances. In the following sections some of these methods and their applications for studying molecular dynamics and local molecular conformation will be discussed. We will focus on examples that show how NMR methods are capable of providing such informations in a model free aproach. In this class of materials we include: semi-crystalline, amorphous, eletroluminescent, and ionic conducting polymers.

\section{INTERNAL SPIN INTERACTIONS}

All the NMR applications for solid-state characterization rely on the behavior of the so-called NMR internal spin interactions. The internal interactions are associated to the couplings of the nuclear spins with, for example, magnetic fields originated from the orbital motion of the electrons induced by the static magnetic field (chemical shift interaction - CS), other nuclear spins through their magnetic dipole moments (dipolar interaction - DD), and (when the nuclear spins are higher than $\frac{1}{2}$ ) the electric field gradients through their electric quadrupole moments (quadrupolar interaction - Q). Using common rotation properties of these internal interactions, in first order approximation the contribution of any interaction to the NMR frequency can be written in a general form as $[1,2]$

$$
\begin{gathered}
\omega=\omega_{\text {iso }}+\omega_{\text {aniso }}(\theta, \phi)= \\
=\gamma B_{0}\left\{R_{\text {iso }}+\frac{\delta}{2}\left(3 \cos ^{2} \theta-1+\eta \sin ^{2} \theta \cos 2 \phi\right)\right\}
\end{gathered}
$$

$R_{i s o}, \delta$, and $\eta$ are respectively the isotropic term, the anisotropy factor, and the asymmetry parameter, which define the tensor representing the nuclear spin interactions. $\theta$ and $\phi$ are the Euler angles by which the laboratory frame (with $z$-axis defined as the main magnetic field, $B_{0}$, direction) can be brought into coincidence with the principal axes system (PAS) in which the interaction tensor is diagonal. Because tensors that represent the dipolar and quadrupolar interactions are traceless, their isotropic contributions are equal to zero, and the only isotropic term different from zero is that of the chemical shift interaction.

The measurement of the tensor parameters $R_{i s o}, \delta$, and $\eta$, as well as the orientation angles are among the main purposes of the Solid-State NMR spectroscopy. In principle, they can be obtained directly from the NMR line shape in the cases where there is a strongly dominant spin interaction and the nuclear spins are NMR equivalents (they are subject to the same internal fields), Fig. 1. However, this is rarely achieved because in real samples the nuclear spins are not NMR equivalents (the internal fields reflect the chemical and local structure of the molecular sites) and they are subjected to more than one NMR interaction. Actually, there are several main factors governing the NMR experiment to access the tensor parameters: the relative intensity of the internal interactions compared to the Zeeman interaction (interaction of the spins with the main magnetic field); the relative intensities of the internal interactions; the manner in which the internal interactions manifest themselves in the NMR spectra; and the natural (molecular motions) or intentional (advanced NMR methods) averaging of the internal interactions in both ordinary and/or 
spin spaces. Thus, for recovering the information about the characteristics of the NMR interactions and their relationship with the local structure and dynamics of materials it is necessary to perform specifically designed experiments. Indeed, the development of NMR methods is itself a research area. A huge number of NMR techniques were and are still being developed, not only to increase the reliability of the current methods, but also to provide new information about the structure, dynamics, and morphology of complex materials in an almost model free approach.

\section{MOLECULAR DYNAMICS}

Solid-state NMR provides powerful methods that allow detailed studies of molecular dynamics occurring over a wide range of frequencies [3]. Fast dynamics $(\sim 100 \mathrm{MHz})$ can be characterized to some extent by NMR relaxation-time measurements [4,5]. Dynamics occurring within the $\mathrm{kHz}$ frequency scale can be studied by line shape analysis [6] or dipolar-chemical shift correlation methods [7]. Slow dynamics $(1 \mathrm{~Hz}-1 \mathrm{kHz})$ can be studied by the so-called Exchange NMR experiments, where relatively slow conformational transitions or segmental reorientations are observed in terms of changes of orientation-dependent NMR frequencies [2]. In the following sections some of these methods and their applications for studying molecular dynamics will be discussed. Because NMR relaxation methods have been extensively reviewed elsewhere and there are many specialized books $[5,8]$ that address this topic, they will not be discussed. We will concentrate our attention on the NMR methods capable of obtaining information about molecular dynamics and conformation of polymers and derivatives.

\section{A. Analysis of motions in the $\mathrm{kHz}$ frequency scale: Line shape analysis and dipolar-chemical shift correlation experiments}

The analysis of the behavior of the NMR line shapes as a function of temperature is one of the most traditional procedures for probing molecular dynamics using Solid-State NMR. Only under static conditions the NMR Hamiltonians can be written as in Eq. 1, which results in the NMR powder patterns shown in Fig. 1. In other words, if there are molecular motions, regular powder patterns will be observed only if its correlation time, $\tau_{c}$, is much longer than the inverse of the anisotropy factor of the spin interaction, i.e., $\delta \tau_{c}>>2 \pi$ (slow exchange regime). At larger motional rates, i.e., smaller $\tau_{c}{ }^{\prime} s$, ( $\delta \tau_{c} \sim 2 \pi$ - intermediate exchange regime) the NMR spectrum is not only defined by the static anisotropic NMR frequency, Eq.1, but also depends on the time scale and geometry of the molecular motion [2]. In the limit where many frequency changes occur during the signal acquisition, $\delta \tau_{c}<<2 \pi$, (fast exchange limit) [2] a fully averaged powder pattern with the shape defined by the characteristics of the molecular motion is observed. The characteristic shape of the spectrum obtained in the fast exchange regime is dictated by the amplitude and geometry of the motion. In general, the NMR line shape in the fast exchange limit can be obtained simply by calculating the effective precession frequency (average frequency) under the anisotropic spin interaction [2]. This effective precession frequency is calculated according to an average tensor that depends on the motion geometry. Because the average tensor is also a second rank tensor, its anisotropy can also be characterized by two parameters namely $\bar{\eta}$ and $\bar{\delta}$.

The dependence of $\bar{\eta}$ and $\bar{\delta}$ with the geometry of the motion is demonstrated in Figs. $2 a$ and $b$, for the cases of chemical shift anisotropy - CSA (for spin $1 / 2$ nuclei) and first order quadrupolar (for spin 1 nuclei) interactions, respectively. Different spectra are observed according to the motional geometry and amplitude. Some general features shown in Fig. 2 are: i) for any uniaxial motion, such as the three-site jump, the resulting average tensor becomes axially symmetric (it has $\bar{\eta}=0$ ); and ii) for isotropic motions the average tensor parameters are $\bar{\eta}=0$ and $\bar{\delta}=0$, i.e., the anisotropy of the spin interaction is fully averaged. In the intermediate exchange regime the line shape also changes according to the correlation time. This is shown in Figs. 2c and d for chemical shift and quadrupolar interactions.

An example of ${ }^{13} \mathrm{C}$ NMR line shape analysis to investigate molecular dynamics can be found in the study of the polymer molecular dynamics in siloxane/poly(ethylene glycol) (PEG) nanocomposites, also called ormolytes [9]. This hybrid system belongs to a family of versatile compounds, classified as di-ureasils, in which polyether-based chains are grafted at both ends to a siliceous backbone through urea functionalities [10]. The polymer chain in ormolytes has the following chemical structure: $\mathrm{R}-\left[\mathrm{OCH}_{2} \mathrm{CH}_{2}\right]_{n} \mathrm{O}-\mathrm{R}$, where $\mathrm{R}=$ $\left(\mathrm{CH}_{2}\right)_{3} \mathrm{NHCONH}\left(\mathrm{CH}_{2}\right)_{3}$. The organic part of the ormolyte is composed of a linker group, $\left(\mathrm{CH}_{2}\right)_{3} \mathrm{NHC} \mathrm{ONH}\left(\mathrm{CH}_{2}\right)_{3}$, and $n$ ethylene glycol repeat units $\left[\mathrm{OCH}_{2} \mathrm{CH}_{2}\right]$ [11]. When doped with Li salts, these solid, transparent, and flexible nanocomposites exhibit a good ionic conductivity at room temperature $\left(\sim 10^{-6} \mathrm{~S} / \mathrm{cm}\right)$. Due to the presence of covalent bonds between the inorganic and organic phases, these hybrids exhibit good chemical stability and mechanical properties. The nomenclature used to describe the composites is $[\mathrm{X}]_{n}[\mathrm{Y}]$, where $\mathrm{X}$ represents the weight percent of polymer, $n$ the polymerization degree, and [Y] the oxygen-to-lithium ratio, respectively.

The possibility of observing separated ${ }^{13} \mathrm{C}$ chemical shift powder patterns is achieved by acquiring the ${ }^{13} \mathrm{C}$ NMR spectrum under high power ${ }^{1} \mathrm{H}$ decoupling (DD) [1]. This method consists on irradiating the ${ }^{1} \mathrm{H}$ nuclei on resonance in order to average out the dipolar interaction between the ${ }^{1} \mathrm{H}$ and ${ }^{13} \mathrm{C}$ nuclei. Besides, the use of the cross-polarization excitation (CP) enhances considerably the ${ }^{13} \mathrm{C}$ nuclei signal, making possible to perform such experiment without the necessity of ${ }^{13} \mathrm{C}$ labeling [2]. The use of standard methods such as crosspolarization and heteronuclear ${ }^{1} \mathrm{H}$ decoupling makes possible to study the dynamics of polymer chains by analyzing the changes in the chemical shift powder patterns as a function of temperature. Fig. 3a (left) shows the ${ }^{13} \mathrm{C}$ line shape temperature dependence for Li doped ormolyte [88] ${ }_{47}[30]$. The spectral lines observed at the highest temperatures were already assigned [9]. The resonances found between 20 to $-20 \mathrm{ppm}$ and 

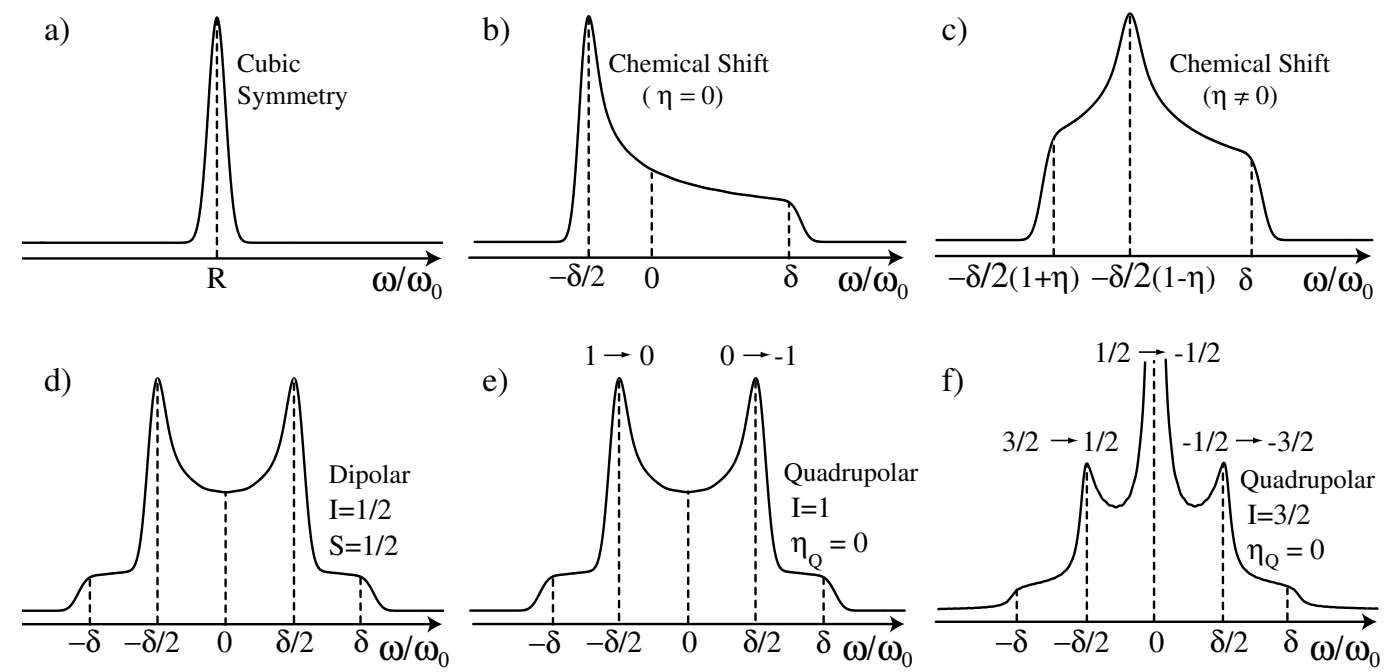

FIG. 1: Calculated NMR spectra of polycrystalline or amorphous samples (powder patterns) corresponding to different NMR interactions: a-c) Chemical Shift under different symmetry conditions; d) Dipolar interaction between two spins 1/2 distant each other by a fixed distance; and e-f) Quadrupolar interaction for spins 1 and 3/2.

above $120 \mathrm{ppm}$ (not shown) spectral regions are attributed to carbons in the linkage group $\left(\mathrm{CH}_{2}\right)_{3} \mathrm{NHCONH}\left(\mathrm{CH}_{2}\right)_{3}$, while the signal observed around $70 \mathrm{ppm}$ to $\left[\mathrm{CH}_{2} \mathrm{CH}_{2} \mathrm{O}\right]$ groups in $\mathrm{PEG}$ chains. In the rigid-lattice regime, at the lowest temperatures, a typical $\left[\mathrm{CH}_{2} \mathrm{CH}_{2} \mathrm{O}\right]$ powder pattern is observed from 35 to $100 \mathrm{ppm}$. In this case, the tensor parameters $\sigma_{i s o}, \delta$, and $\eta$ for the ${ }^{13} \mathrm{C}$ nuclei in the $\left[\mathrm{CH}_{2} \mathrm{CH}_{2} \mathrm{O}\right]$ groups can be obtained directly from the powder pattern: $\sigma_{i s o}=69 \mathrm{ppm}, \delta=33 \mathrm{ppm}$, and $\eta=0.3$. The analysis of the ${ }^{13} \mathrm{C}$ line shapes versus temperature provides information about molecular motion in the $\mu$ s to ms time scale. This is achieved by simulating the evolution of the ${ }^{13} \mathrm{C}$ magnetization belonging to different sites (different orientation of the molecules in respect to the $B_{0}$ field) under the influence of the molecular motions [2]. However, for performing such calculations it is necessary to know the geometry of the motion. In the specific case of the ormolytes, 2D Exchange measurements suggest that the isotropic rotational diffusion model would be appropriated for describing the dynamics of the PEG chains [9]. Thus, comparing the simulated spectra with the corresponding experimental ones, the temperature dependence of the mean correlation time is obtained and the apparent activation energy of the motions is extracted. Alternatively, a different analysis for obtaining the activation energy can be performed. It consists of measuring the ${ }^{13} \mathrm{C}$ line width as a function of temperature, Fig. 3a (right). Because the line width versus correlation time can be simulated using the above method, from the comparison of these simulated curves with the temperature dependence of the line width it is possible to obtain the correlation time-temperatue correspondence, which provides the activation energy of the motion. This is particularly suitable in the case of ormolytes, because the partial superposition of the signals arising from the $\left[\mathrm{CH}_{2} \mathrm{CH}_{2} \mathrm{O}\right]$ and linker groups makes difficult to compare the simulated and experimental spectral patterns. Experimental as well as theoretical line width vs. temperature are shown in Fig. 3a (right) for the ormolyte [88 $]_{47}[30]$. The extracted apparent activation energy was $(53 \pm 2) \mathrm{kJ} / \mathrm{mol}$, which is similar to the activation energy found for the $\mathrm{Li}^{+}$motion [12]. Notice that the line widths were taken at $2 / 3$ of the maximum intensity. This avoids the contribution of the linker signal and also produces a curve, which has a pronounced maximum that can be taken as a reference temperature for glass transition.

The use of ${ }^{1} \mathrm{H}$ line shapes is also a traditional method for studying molecular dynamics. The ${ }^{1} \mathrm{H}$ NMR spectrum of rigid materials is characterized by broad lines, usually with gaussian or lorentzian shapes due to the strong coupling between the ${ }^{1} \mathrm{H}$ nuclei. As in the case of any other anisotropic interaction, molecular motion can induce the averaging of the ${ }^{1} \mathrm{H}-{ }^{1} \mathrm{H}$ dipolar interaction, reducing the line widths. Monitoring the line width as a function of temperature and using appropriate models, information about correlation times and activation energies of molecular motions can be obtained $[12,13]$. These studies, which can also be performed for other nuclei, are useful for the characterization of materials like solid polymer electrolytes, where the line width and $T_{1}$ relaxation of the charge carriers (usually $\mathrm{Li}, \mathrm{Be}, \mathrm{Ag}$, or $\mathrm{Cs}$ ), provide information about their mobility $[12,13]$. However, due to the lack of spectral resolution in the ${ }^{1} \mathrm{H}$ spectra, these studies have been limited to the cases where no local information about molecular motions are necessary. This is overcome by using the individual $\mathrm{C}-\mathrm{H}$ dipolar coupling as a probe for the molecular motion, which is achieved in a class of experiments based on Dipolar-Chemical Shift Correlation [6,14]. These experiments can be performed under magic-angle spinning (MAS) [2]. The concept of MAS is to induce a mechanical rotation of the sample in such a way that the orientation dependence of the internal NMR interactions is fully or partially averaged. Usually, in experiments on rare nuclei like ${ }^{13} \mathrm{C}$, both heteronuclear ${ }^{1} \mathrm{H}$ decoupling and MAS are applied simultaneously to achieve reasonably narrow lines by 
a) CSA $\quad \delta_{\mathrm{CSA}}=83 \mathrm{ppm}$
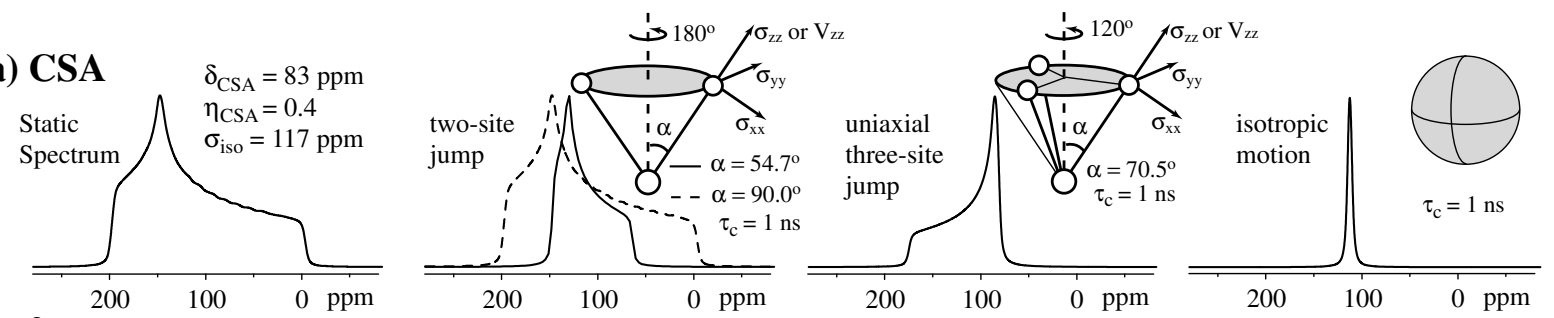

b) ${ }^{2} \mathbf{H}$
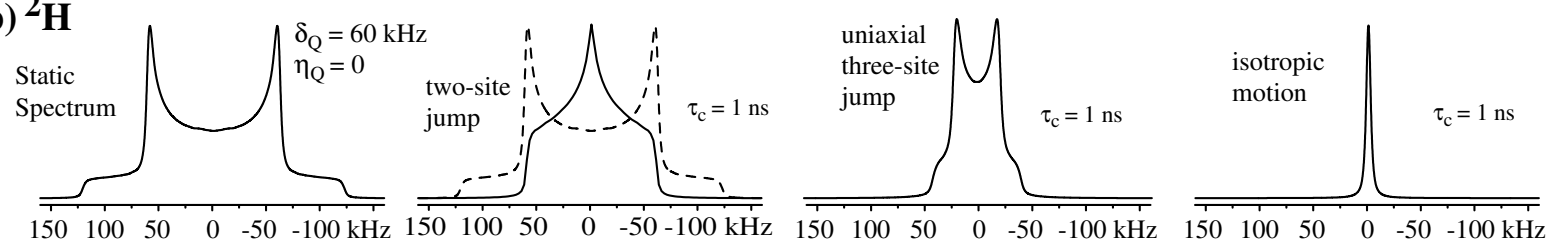

c) CSA
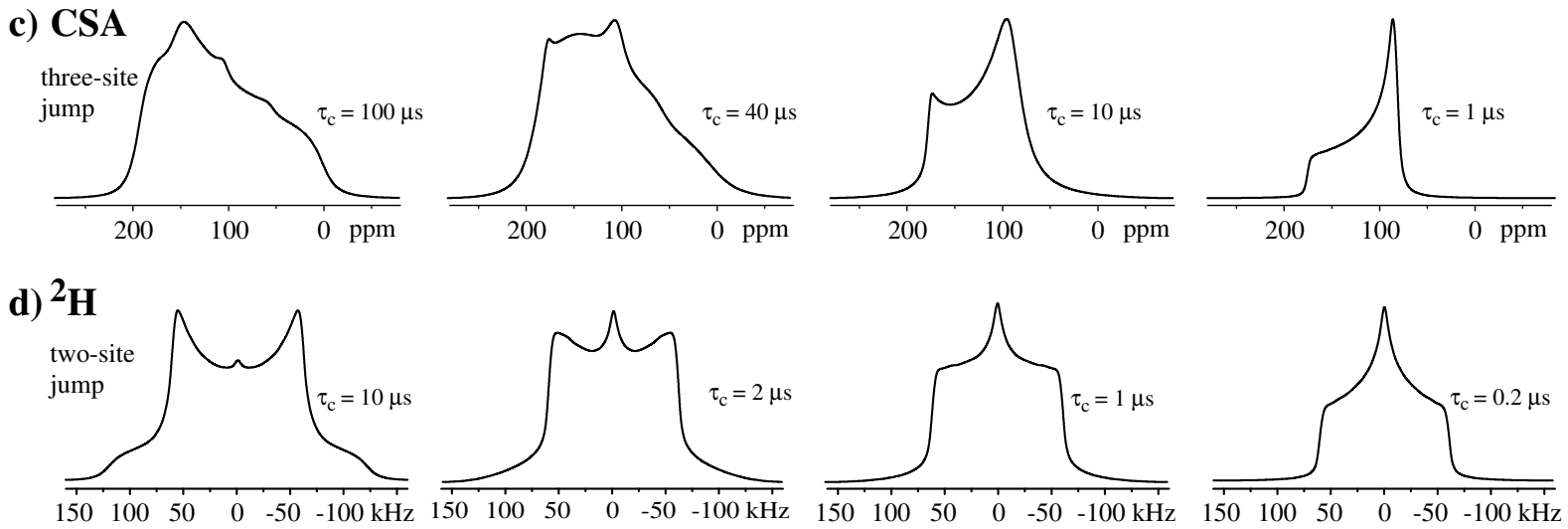

FIG. 2: Series of CSA and quadrupolar $\left({ }^{2} \mathrm{H}\right)$ powder patterns simulated for different kinds of molecular motions. a) CSA powder patterns. b) ${ }^{2} \mathrm{H}$ powder patterns. c) CSA powder pattern for three-site jump with $\alpha=70.5^{\circ}$ (methyl rotations) and different correlation times. d) ${ }^{2} \mathrm{H}$ Powder pattern for two-site jump with $\alpha=54.7^{\circ}$ and different correlation times.

coherent averaging of the heteronuclear dipolar interaction and chemical shift anisotropy. As a result, a ${ }^{13} \mathrm{C}$ spectrum acquired using a combination of cross-polarization, ${ }^{1} \mathrm{H}$ decoupling, and MAS (CP/MAS) contains sharp lines associated with the different chemical groups in the sample. One example of Chemical Shift Dipolar correlation experiment for studying molecular dynamics is the two-dimensional LeeGoldburg cross-polarization (2D LG-CP) [7]. In this method, the cross-polarization time is incremented to produce a $2 \mathrm{D}$ spectrum that contains the ${ }^{13} \mathrm{C}$ high-resolution NMR spectrum along one spectral dimension and the corresponding scaled ${ }^{13} \mathrm{C}-{ }^{1} \mathrm{H}$ dipolar powder pattern spectrum along the other one (see pulse sequence shown in Fig. 3b). To achieve that the use of a cross-polarization procedure combined with a homonuclear ${ }^{1} \mathrm{H}-{ }^{1} \mathrm{H}$ decoupling method (Lee-Goldburg crosspolarization) is crucial. This is so because the high ${ }^{1} \mathrm{H}$ natural abundance and the strong ${ }^{1} \mathrm{H}-{ }^{1} \mathrm{H}$ dipolar coupling makes the ${ }^{1} \mathrm{H}$ magnetization transfer $\left({ }^{1} \mathrm{H}\right.$ spin-diffusion) during the cross-polarization period very effective [15]. This destroys the local character of the cross-polarization, and must be avoided if information about local motions is necessary. By using Lee-Goldburg cross-polarization, the ${ }^{1} \mathrm{H}$ spin-diffusion is quenched and the ${ }^{13} \mathrm{C}-{ }^{1} \mathrm{H}$ dipolar powder spectrum of each chemical group can be measured in the $2 \mathrm{D}$ spectrum.
Figure $3 \mathrm{c}$ shows the ${ }^{13} \mathrm{C}$ CP/MAS spectrum of the electroluminescent polymer poly(2-methoxy,5-(2'-ethylhexyloxy),p-phenylenevinylene), MEH-PPV, with the corresponding line assignments. MEH-PPV is an electroluminescent active polymer [16] where the molecular chain dynamics is directly related with its luminescent properties [17]. Fig. $3 \mathrm{~d}$ shows the ${ }^{13} \mathrm{C} 2 \mathrm{D}$ LG-CP spectrum in the side group region of MEH-PPV. As discussed before, the spectrum allows obtaining ${ }^{13} \mathrm{C}-{ }^{1} \mathrm{H}$ dipolar patterns for each different carbon in the side group, as shown by the spectrum traces of Fig. 3d. As a general trend, the narrowing of the spectrum due to the averaging of the $\mathrm{C}-\mathrm{H}$ coupling is readily observed, suggesting that the mobility might be different at each position in the side group. More specifically, the Pake doublet is well defined for $\mathrm{CH}_{2}$ carbons 12 and 13, while it is almost averaged out for $\mathrm{CH}_{2}$ carbons 14 and 16. This shows that there is a difference in the mobility even for chemical groups separated by only one bond. Besides that, the spectral shape is also considerably changed and, because the 2D LG-CP pattern is very sensitive to changes in the motional amplitude, this shows that the motional amplitude is increasing from carbons 11 to 14 .

Another useful chemical shift-dipolar correlation experiment for studying molecular dynamics in organic materials is the 1D DIPSHIFT method [18]. Simply put, the 1D DIP- 
SHIFT pulse sequence imposes a signal modulation to each ${ }^{13} \mathrm{C}$ nucleus according to its dipolar coupling to the neighbor ${ }^{1} \mathrm{H}$ nucleus $\left({ }^{13} \mathrm{C}-{ }^{1} \mathrm{H}\right.$ coupling). As discussed before, motions with correlation times shorter than $\sim 10 \mu$ s scale down the magnetic dipolar coupling between ${ }^{1} \mathrm{H}$ and ${ }^{13} \mathrm{C}$, making possible to distinguish rigid from mobile segments and also to estimate the amplitude of the motion by specific modeling. The pulse sequence used in the 1D DIPSHIFT experiments is shown in Fig. 4a. After the excitation of the ${ }^{13} \mathrm{C} \mathrm{nu}-$ clei, usually using the cross-polarization procedure, the ${ }^{13} \mathrm{C}$ coherences evolve under the effect of the isotropic chemical shift and ${ }^{13} \mathrm{C}-{ }^{1} \mathrm{H}$ coupling during the period $t_{1}$, which varies from zero to one rotor period $t_{r}$. This is achieved by applying a ${ }^{1} \mathrm{H}-{ }^{1} \mathrm{H}$ Frequency-Switched Lee-Goldburg (FSLG) decoupling sequence [19] during this period. After this first evolution period, the transverse ${ }^{13} \mathrm{C}$ magnetizations are modulated by the corresponding ${ }^{13} \mathrm{C}-{ }^{1} \mathrm{H}$ couplings scaled by a factor that depends on the pulse sequence used for ${ }^{1} \mathrm{H}-{ }^{1} \mathrm{H}$ homonuclear decoupling [19]. After $t_{1},{ }^{13} \mathrm{C}-{ }^{1} \mathrm{H}$ heteronuclear decoupling is applied for the remaining $t_{r}-t_{1}$ period and exactly at one rotor period a $\pi$ pulse is applied in the ${ }^{13} \mathrm{C}$ channel. The application of the $\pi$ pulse produces an echo at $2 t_{r}$, due to the refocusing of the isotropic chemical shift, with amplitude modulated by $\left\langle\cos \left(2 \pi v_{C H} t_{1}\right)\right\rangle$, where $v_{C H}=\frac{\delta_{C H}}{2}\left(3 \cos ^{2} \theta-1\right)$, $\delta_{C H}\left(\sim c t e / r_{C H}^{3}\right)$ is the anisotropy factor for the heteronuclear ${ }^{13} \mathrm{C}-{ }^{1} \mathrm{H}$ dipolar interaction, and $\theta$ is the angle between the $\mathrm{CH}$ bond and the static magnetic field $B_{0}$. In summary, the experiment basically produces an echo where the chemical shift interaction is refocused and the evolution due to the C-H dipolar coupling is retained. Measuring the signal amplitude as a function of $t_{1}$, a typical curve whose shape depends on $v_{C H}$ is obtained, Fig. 4b, allowing estimating the dipolar anisotropy factor by fitting the experimental data by numerically simulated curves for a given $\delta_{C H}$. If molecular motions with correlation times shorter than $\sim 10 \mu$ s are present, the strength of the anisotropic dipolar coupling is partially averaged and motionally averaged anisotropic coupling $\overline{\mathrm{v}}_{C H}$ is measured in DIPSHIFT experiments. This motionally averaged dipolar coupling divided by its rigid limit value defines the molecular order parameter, $S$, of the $\mathrm{CH}_{n}$ bonds, i.e., the order parameter associated to each chemical group.

Using 1D DIPSHIFT, molecular order parameters were measured for each chemical group along the MEH-PPV polymer chain, Figs. 4c and d [20,21]. These results show that carbons in the polymer main chain have temperature independent order parameters of approximately 1 . The order parameter for methoxy carbons (9) is also temperature independent and the obtained $S$ value is $0.33 \pm 0.02$, which is characteristic of $\mathrm{CH}_{3}$ groups executing axial rotations around its $\mathrm{C} 3$-axis. For the $\mathrm{CH}$ carbon 11 in the side-group, the order parameter decreases monotonically with temperature, indicating a gain of mobility. $\mathrm{CH}_{3}$ carbons in the side-groups (15 and 17) also exhibit a $S$ decay as a function of temperature and in this case it is smaller than 0.33. This is a consequence of the fact that these $\mathrm{CH}_{3}$ groups located in the side-chain follow the motion of the side-groups in addition to the axial rotations. Another interesting issue is the temperature dependence of dipolar coupling for $\mathrm{CH}_{2}$ groups $10,12,13,14$, and 16 along the side a)
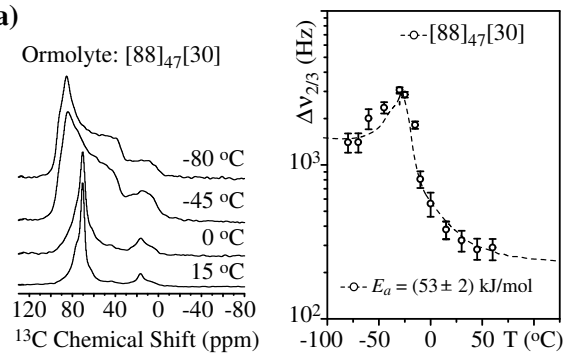

b)
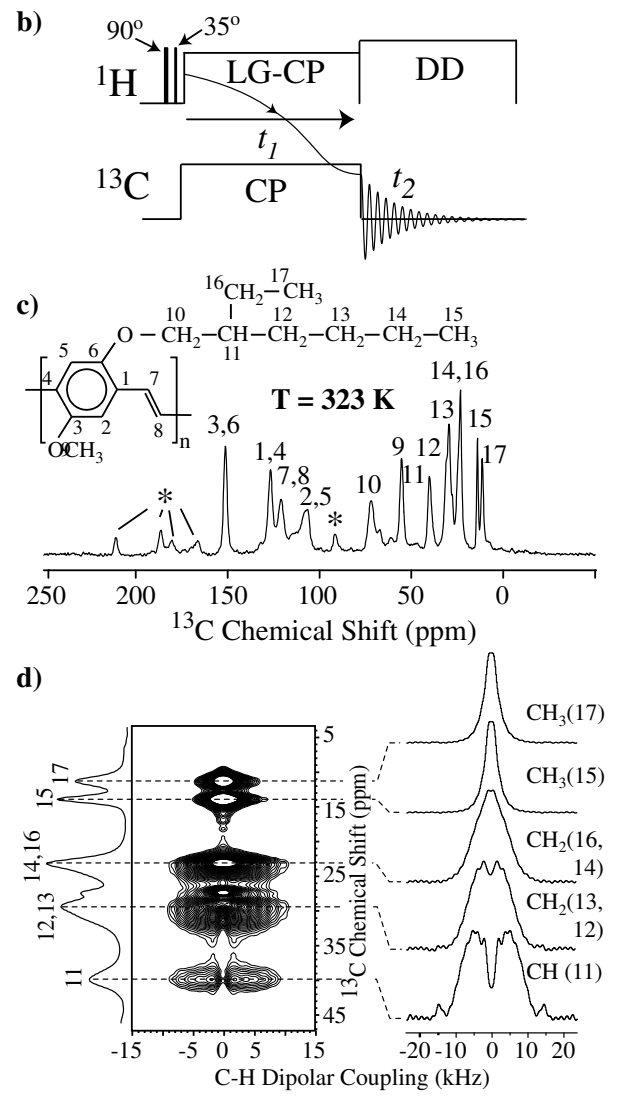

FIG. 3: a) CSA powder pattern (left) and line width at $2 / 3$ of the maximum intensity measured as a function of temperature (right) for the ormolyte [88 $]_{47}[30]$. The dashed lines represent simulated curves based on the isotropic rotational diffusion model. b) Pulse sequence for $2 \mathrm{D}$ LG-CP experiments. c) ${ }^{13} \mathrm{C} \mathrm{CP} / \mathrm{MAS}$ spectrum of the photoluminescent polymer MEH-PPV (The symbols * indicate spinning sidebands). d) ${ }^{13} \mathrm{C} 2 \mathrm{D}$ LG-CP spectrum of MEH-PPV. Only the spectral region corresponding to the side-groups is shown. (Adapted with permission from reference [21] - Copyright 2005 American Physical Society)

chain. Because the dipolar coupling for $\mathrm{CH}_{2}$ carbon $10, \mathrm{v}_{\text {dip }}^{C_{10}}$, was found to be temperature independent, the order parameters were calculated using the dipolar coupling of this group as a reference, $S=\left\langle v_{d i p} / v_{d i p}^{C_{10}}\right\rangle$, for the side chain carbons. The temperature dependence of the order parameter for all the side chain $\mathrm{CH}_{2}$ groups is shown in Fig. 4d. The order parameters for side chain carbons $(12,13,14$, and 16) decrease as a function of temperature.

Figure 4e shows MEH-PPV photoluminescence (PL) spec- 


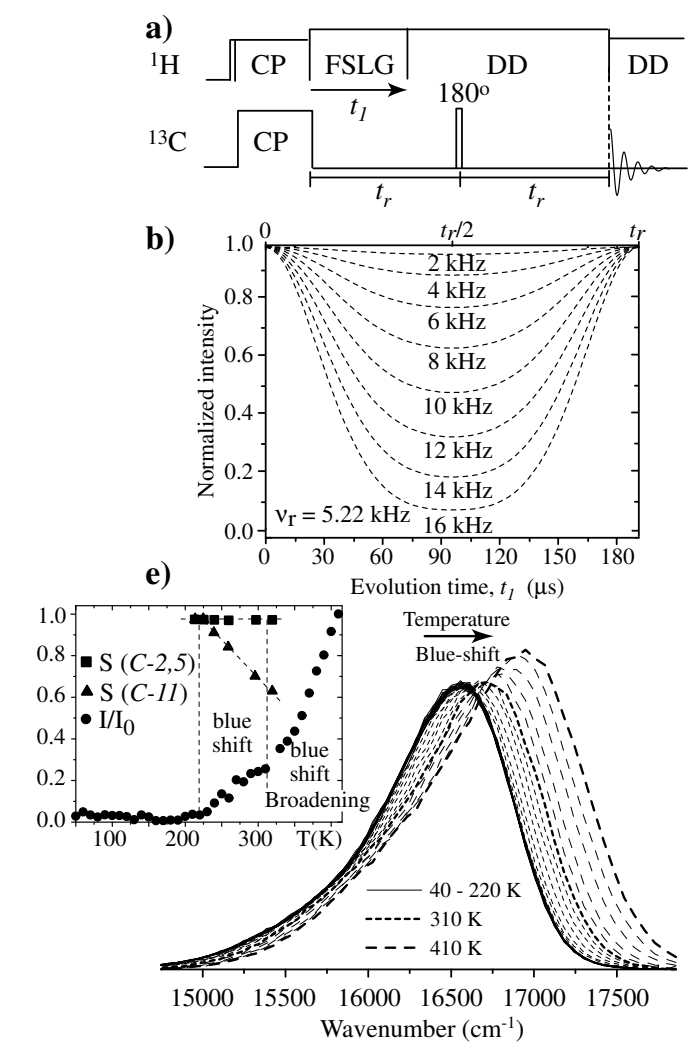

c)

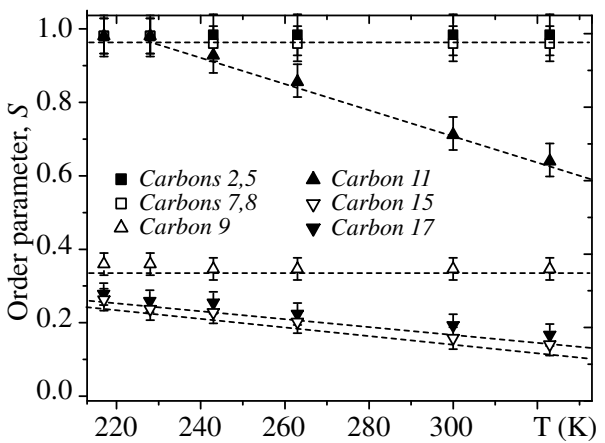

d)

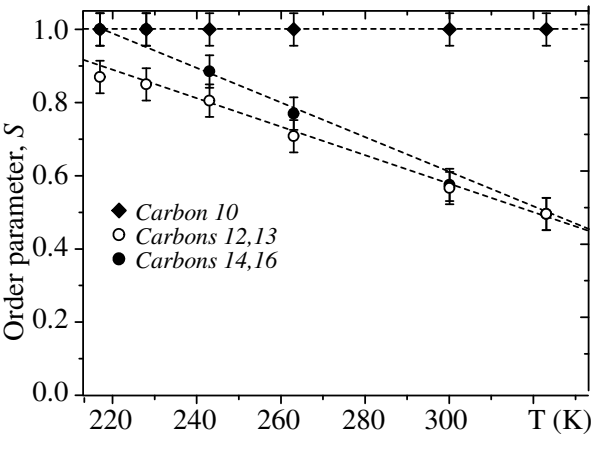

FIG. 4: a) Pulse sequence used in 1D DIPSHIFT experiments. b) Typical $t_{1}$-dependence of 1D DIPSHIFT curves for different CH couplings $\left(\delta_{C H}\right.$ in $\left.\mathrm{kHz}\right)$. c) Order parameters for $\mathrm{CH}$ carbons in MEH-PPV obtained from 1D DIPSHIFT experiments. d) Same as c) for $\mathrm{CH}_{2}$ carbons. e) Photoluminescence spectrum of MEH-PPV as function of temperature. (Adapted with permission from reference [21] - Copyright 2005 American Physical Society)

tra at several temperatures, from 40 to 410 K.[20] As it can be seen, there are three regimes in this series of spectra. From 40 to $220 \mathrm{~K}$ the spectral characteristics remain mostly unchanged; between 220 and $320 \mathrm{~K}$ width and spectral intensity remain constant and a spectral blue-shift is observed; above $320 \mathrm{~K}$, besides blue-shift, width and spectral intensity increase continuously. Analyzing the order parameters for side chain carbons $(11,12,13,14$, and 16) shown in Figs. 4c and d, one can observe that they are about 1 at temperatures lower than $\sim 230 \mathrm{~K}$ and decrease for higher temperatures. Because the decrease of the order parameter is strictly related with the increase of the amplitude of the polymer chain motion, it is apparent the correlation between the PL changes and the onset of side group motion above $\sim 220 \mathrm{~K}$ [22]. Therefore, the modifications in the PL spectra, which occur just above 220 $\mathrm{K}$, might be correlated to the change in the microenvironment due to the onset of the side group motion, which produces regions where interchain interactions are less effective than in the frozen state. This shows that the secondary relaxation occurring in side chain is directly related with the processes that affect the photophysics of MEH-PPV [20].

\section{B. Analysis of motions in the $\mathrm{Hz}-\mathrm{kHz}$ frequency scale: Exchange NMR experiments}

\section{1. $2 D$ Exchange NMR}

Exchange NMR experiments provide the most specific information about slow motions $\left(\tau_{C} \sim m s-s\right)$ in organic materials. In these experiments molecular reorientations on the milliseconds to seconds time scale (that do not change the powder spectrum) are observed in terms of changes in the orientationdependent NMR frequencies. Thus, using these techniques, details of slow molecular reorientations such as correlation times and their distributions, reorientation-angle distributions, orientational memory, rate memory, information on the existence of dynamic heterogeneities, and their sizes, can be directly probed $[2,23,24]$. Among the large variety of exchange experiments that can be performed, the 2D Exchange experiment has been extensively applied to study dynamics of several different systems, using ${ }^{13} \mathrm{C}$ or ${ }^{2} \mathrm{H}$ as probe nuclei for the molecular rotations [25-28]. Fig. 5a shows the pulse sequence for the static 2D Exchange experiment. In this experiment the orientation dependent NMR frequencies are monitored before $\left(\omega_{1}\right)$ and after $\left(\omega_{2}\right)$ a mixing time $t_{m}$, when molecular reorientations can occur. The resulting spectrum is a $2 \mathrm{D}$ pattern that correlates the NMR frequencies experienced by the nu- 
a)

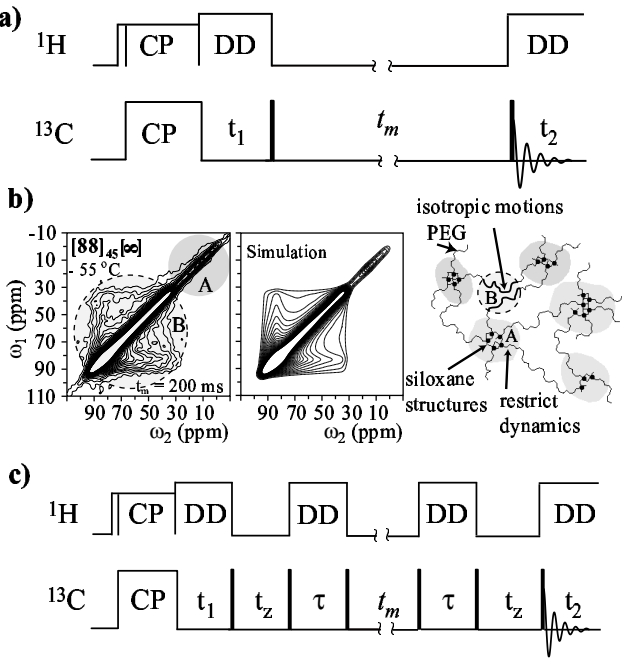

d)
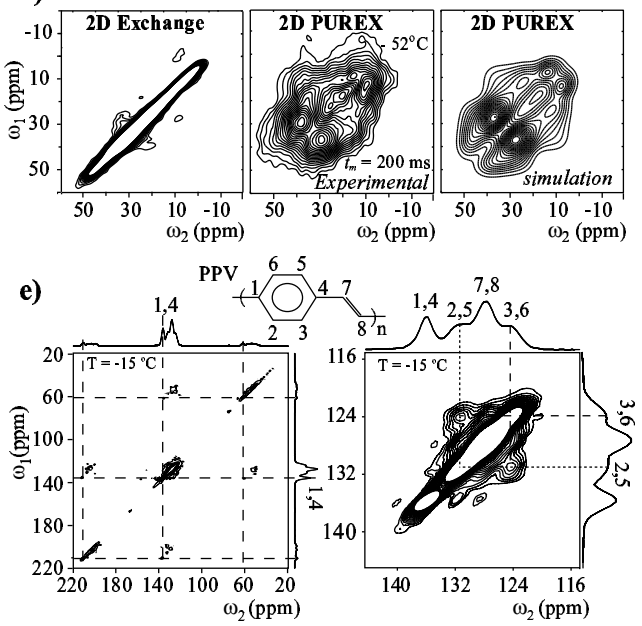

FIG. 5: a) Pulse sequence used in 2D Exchange NMR experiments. $\pi / 2$ pulses are in black. b) ${ }^{13} \mathrm{C} 2 \mathrm{D}$ Exchange spectrum of the ormolyte $\left[{ }^{88}\right]_{45}[\infty]$. Left: experimental spectrum; Middle: Simulation using the isotropic rotational diffusion model; Right: schematic representation of the motions in different regions of ormolytes. c) Pulse sequence for the 2D PUREX experiment. d) ${ }^{13} \mathrm{C} 2 \mathrm{D}$ spectra of the isotatic poly(1-butene) semi-crystalline polymer. Left: Regular 2D Exchange spectrum; Middle: Experimental 2D PUREX spectrum; Right: 2D PUREX Simulation using the isotropic rotational diffusion model. e) 2D MAS Exchange spectrum of the electroluminescent polymer poly ( $p$-vinylene phenylene) (PPV). (Adapted with permission from reference [3] - Copyright 2005 Elsevier, from reference [9] - Copyright 2005 American Chemical Society, and from reference [31] - Copyright 2005 American Institute of Physics)

clear spins during the periods $t_{1}$ and $t_{2}$. If no reorientations occur during $t_{m}, \omega_{1}=\omega_{2}$, the $2 \mathrm{D}$ spectrum is purely diagonal. In contrast, if molecular reorientations occur during $t_{m}$, off diagonal intensities are observed. Because the changes in the NMR frequency during $t_{m}$ is proportional to the molecular rotation angle, the shape of the 2D spectrum, $S\left(\omega_{1}, \omega_{2}, t_{m}\right)$, strongly depends on the motional amplitude. The $2 \mathrm{D}$ spectrum can be interpreted as a map of reorientation probabilities, i.e., it represents the probability density of finding a segment

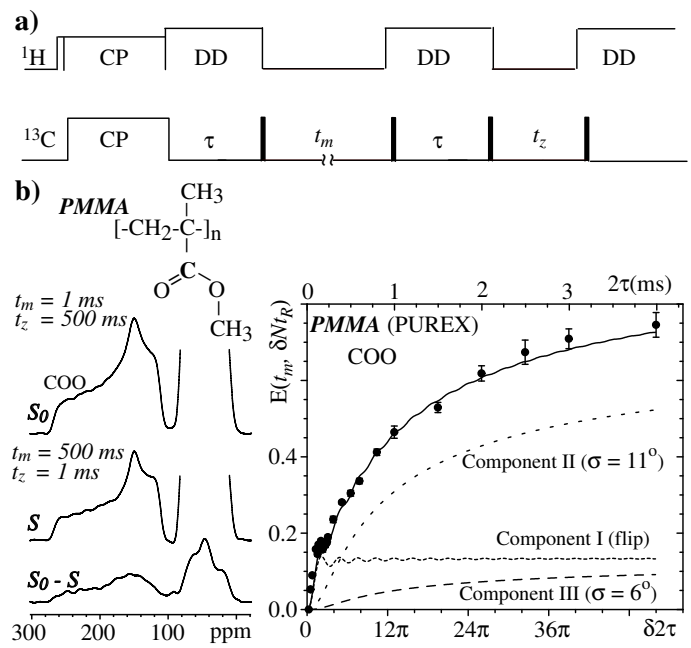

c)
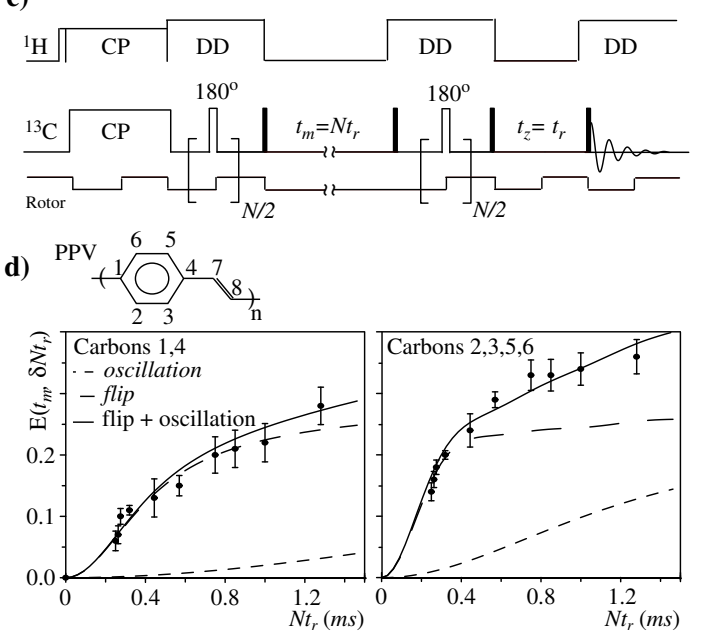

FIG. 6: a) Pulse sequence for 1D Pure Exchange NMR experiments (1D PUREX). b) Left: Set of 1D PUREX spectra for poly(methyl methacrylate) (PMMA). Right: 1D PUREX $E\left(t_{m}, \delta \tau_{C S A}\right)$ vs. $\tau_{C S A}(=2 \tau)$ obtained for PMMA. c) Pulse sequence for CenterbandOnly detection of Exchange Experiments (CODEX). $\pi / 2$ pulses are in black. d) $\operatorname{CODEX} E\left(t_{m}, \delta N t_{r}\right)$ vs. $N t_{r}$ curves for protonated and non-protonated carbons of PPV.(Adapted with permission from reference [31] - Copyright 2005 American Institute of Physics and from reference [43] - Copyright 2005 Springer)

with frequency $\omega_{1}$ before and $\omega_{2}$ after $t_{m}$. Because these probabilities are dictated by the correlation time and the geometry of the motions, the 2D Exchange pattern is sensitive to both of these features. If the molecular motion involves only one or a few reorientation angles, $\beta_{R}$, the reorientation angle can be directly obtained from the experimental spectrum in a modelfree approach [2]. On the other hand, if the molecular motion does not involve only discrete jumps, the geometry of the molecular rotations is characterized by a reorientation angle distribution $\mathrm{R}\left(\beta_{R}, t_{m} / \tau_{c}\right)$. In complex systems rarely the molecular rotations occur with a single correlation time. Instead, it involves a distribution of correlation times $\mathrm{g}\left(\tau_{c}\right)$. In these cases, the 2D Exchange spectrum can be viewed as a superposition of spectra, each one corresponding to a single reorien- 
tation angle and correlation time, $S_{\beta_{R}}\left(\omega_{1}, \omega_{2}, t_{m}\right)$, weighted by the distributions of reorientation angles and correlation times,

$$
\begin{gathered}
S\left(\omega_{1}, \omega_{2}, t_{m}\right)= \\
=\int_{0^{\circ}}^{90^{\circ}} \int_{0}^{\infty} R\left(\beta_{R}, \frac{t_{m}}{\tau_{c}}\right) g\left(\tau_{c}\right) S_{\beta_{R}}\left(\omega_{1}, \omega_{2}, t_{m}\right) d \tau_{c} d \beta_{R}
\end{gathered}
$$

An example of the ${ }^{13} \mathrm{C}$ 2D Exchange NMR experiment applied to investigate the polymer molecular dynamics in siloxane / poly(ethylene glycol) (PEG) nanocomposites is shown in Fig. 5b [9]. It shows the ${ }^{13} \mathrm{C} 2 \mathrm{D}$ Exchange spectrum for the non-doped ormolyte, $[88]_{45}[\infty]$. The spectrum clearly shows two distinct regions. In the spectral region A the NMR signal is exclusively attributed to ${ }^{13} \mathrm{C}$ nuclei in the linkage group, see Fig. 5b (right). The observed exchange pattern is fully diagonal, indicating that these groups do not move in the $m s-s$ time scale. On the other hand, in region B a featureless off-diagonal exchange pattern is observed, indicating that the motion of the $\left[\mathrm{CH}_{2} \mathrm{CH}_{2} \mathrm{O}\right]$ groups does not involve defined jump angles. Fig. $5 \mathrm{~b}$ is a clear model free picture of the strong hindrance to the molecular motion due the siloxane structure, since it directly reveals the distinct dynamic behavior of segments near the siloxane structures. The experimental spectral shape can be well reproduced, Fig. $5 \mathrm{~b}$ (middle), by a simulated pattern that considers $\mathrm{R}\left(\beta_{R}, t_{m} / \tau_{c}\right)$ and $\mathrm{g}\left(\tau_{c}\right)$ corresponding to a small step isotropic rotational diffusion of the polymer chains in the region $\mathrm{B}$ and rigid segments in the region $\mathrm{A}$.

Despite being a widely used method, it is difficult to use 2D Exchange NMR in cases where only a small fraction of the segments take part in the molecular motions. In these cases the strong and sharp diagonal ridge (due to the non-moving segments) overwhelms the weak and broad ridges from the moving groups. This is particularly relevant for small angle reorientations that produce exchange intensities concentrated near the diagonal of the 2D spectrum. To overcome this difficulty, a modified version of the 2D Exchange experiment called 2D-Pure Exchange (2D PUREX) has been developed [29]. In these experiments, extra pulses with adequate phase cycling, extra evolution periods, and spectral subtraction are used together to produce a 2D Exchange spectrum modulated by the term $\sin ^{2}\left(\left[\omega_{1}-\omega_{2}\right] \tau / 2\right)$, where $\tau$ is the extra evolution period added to the pulse sequence, see Fig. 5c. Because of the $\sin ^{2}\left(\left[\omega_{1}-\omega_{2}\right] \tau / 2\right)$ modulation segments that do not reorient during $t_{m}, \omega_{1}=\omega_{2}$, do not contribute to the exchange signal and only segments reorienting on the milliseconds to seconds time scale appear in the resulting $2 \mathrm{D}$ spectrum. One example of the 2D PUREX performance is shown in Fig. 5d, where the technique was applied to study the crystal form I of the semicrystalline polymer isotactic poly(1-butene), $\left(\left[\mathrm{CH}_{2}-\right.\right.$ $\left.\mathrm{CH}-\mathrm{R}-]_{n}-\mathrm{PPB} 1\right)$, at a temperature slightly above its glass transition [29]. The regular 2D Exchange spectrum, Fig. 5d (left), shows a pronounced diagonal ridge that arises from the rigid crystalline portion, and an almost imperceptive off-diagonal pattern, from the mobile amorphous region. In contrast, in the 2D PUREX spectrum the off-diagonal intensity characteristic of diffusive motions is clearly observed, while the diagonal ridge is suppressed, Fig. 5d (middle). The simulation of the 2D PUREX patterns using the isotropic rotation diffusion model reveals the good reliability of the method, Fig. $5 \mathrm{~d}$ (right).

Another important variant of the 2D Exchange experiment is the so-called rotor synchronized 2D MAS Exchange technique. This method was initially proposed by Veeman et al.,[30] who introduced the idea of "rotor synchronization" of pulse sequences when using MAS. This procedure makes sure that the $t_{m}$ is an integer number of rotation periods, $t_{m}$ $=N t_{r}$, so that the precession of the magnetization resumes at the same rotor orientation found at the beginning of the mixing time. Otherwise, the reorientations of segments relative to the external field that occurred due to the macroscopic rotation of the sample (MAS) would overwhelm the more subtle effects of intrinsic segmental reorientations. The principle of the experiment is the same as the conventional exchange NMR methods for non-rotating solids discussed above, i.e., it correlates the anisotropic NMR frequencies in two different periods of time $\left(t_{1}\right.$ and $\left.t_{2}\right)$ separated by a synchronized mixing time $t_{m}$ [26]. The appearance of off-diagonal peaks in the two-dimensional spectra is directly associated with changes in the orientation-dependent NMR frequencies. When performed under slow spinning frequencies, relatively to the anisotropy of the interaction used to probe the molecular motion, the spectrum consists of a $2 \mathrm{D}$ sideband pattern [30], which depends on the characteristics of the slow molecular reorientations. Because isotropic chemical shifts may also change according to the local conformation of a given molecular segment, MAS exchange is also a powerful tool for studying conformational dynamics. In the absence of molecular motion during $t_{m}$, a normal MAS sideband pattern along the diagonal is observed. In the case of molecular motions that only change the orientation of the chemical shift tensor principal axis, cross peaks, labeled as auto-cross peaks, appear at $\left(\omega_{1}, \omega_{2}\right)=\left(\omega_{i s o}^{i}+M \omega_{R}, \omega_{i s o}^{i}+N \omega_{R}\right)$, where $\omega_{i s o}^{i}$ is the isotropic chemical-shift frequency of a nucleus in a given molecular site $i ; \omega_{R}$ is the spinning frequency, and $M$ and $N$ are the orders of the spinning sidebands. On the other hand, if the exchange process involves a change in the isotropic chemical shift, cross peaks, labeled as hetero-cross peaks, appear at $\left(\omega_{1}, \omega_{2}\right)=\left(\omega_{i s o}^{i}+M \omega_{R}, \omega_{i s o}^{j}+N \omega_{R}\right)$, where $i$ and $j$ indicate the change in the isotropic chemical shift of a given nucleus. Thus, if there is an exchange process that changes the isotropic chemical shift, cross-peaks linking isotropic chemical shift frequencies appear in the centerband $(M=N=0)$ even if no side bands are observed, i.e., at high spinning frequencies.

An example of motion that produces changes in the isotropic chemical shifts is the ring-flip in poly( $p$-vinylene phenylene) (PPV) [31]. PPV has also been subject of great interest in the latest years due to its electroluminescent and semiconducting properties, and applications in optoelectronic devices. Vibrational motions, such as phenylene ring flips, strongly couple to the electronic structure and accompanies the relaxation of the initial photoexcitation on the excited 
state, making important to understand details of such dynamic process. Fig. 5e (left) shows the 2D MAS Exchange of PPV acquired at $258 \mathrm{~K}$ and spinning frequency of $7.5 \mathrm{kHz}$. Auto cross-peaks are clearly observed, indicating the presence of slow moving groups in the the aromatic rings of PPV. Besides that, the spectrum also clearly shows the presence of hetero-cross peaks linking the 2,5 and the 3,6 lines, indicating the presence of an exchange process where the isotropic chemical shifts of the 2,5 and 3,6 carbons are interchanged, Fig. 5e (right). Since the isotropic chemical shifts of carbons 2,5 and 3,6 depend on the conformation of these segments relative to the vinylene carbon, the interchange between the isotropic chemical shifts can be attributed to a $180^{\circ}$ rotation of the phenylene rings around the 1,4 axis. Some other examples of applications of rotor synchronized 2D MAS Exchange NMR are the study of conformational dynamics in amorphous and semicrystalline polymers, liquid crystals, and the study of tautomerism and molecular dynamics in molecular crystals $[25,32,33]$.

\section{ID Exchange NMR}

Beyond the 2D Exchange NMR methods, there is a class of one dimensional (1D) techniques that have been used for extracting two- and multiple-time correlation functions of rotational molecular motions occurring on the milliseconds to seconds time-scale [34-39]. In particular, the Stimulated-Echo experiment (STE) [37] has been widely employed for studying the rates, amplitudes, and heterogeneities of slow motions in organic and inorganic solids. However, the use of this technique for studying motions in chemically complex systems suffers from the same problem as the regular 2D Exchange experiment, i.e., there is no specific site resolution, and it is very difficult to characterize the exchange intensity if only a small fraction of the molecular segments takes part in the molecular motion. To overcome the later problem a 1D Pure Exchange version of the stimulated-echo technique was developed $[29,40]$. This technique, named 1D Pure Exchange NMR (1D PUREX), suppresses the signals of immobile molecules and thus provides spectra selectively from segments that reorient during the mixing time. Thus, the amplitude and time scale of slow motions can be determined even for a small fraction of mobile segments. The pulse sequence for the 1D PUREX NMR, Fig. 6a, experiment is obtained from the 2D PUREX pulse sequence simply by removing the $t_{1}$ evolution and the first $z$-period (the two $90^{\circ}$ pulses flanking the $t_{z}$ period, Fig. 5c). Hence, after excitation the spins immediately start evolving under their chemical-shift anisotropies (CSA) during the period $\tau$. The magnetization is then stored along the $z$-direction, so that it does not precess or dephase during a long mixing time, $t_{m}$. In the absence of slow motions during $t_{m}$, the CSA is refocused after a read-out pulse and another evolution period $\tau$ (total evolution time of $\tau_{C S A}=2 \tau$ ). At this point, the magnetization is stored again along the $z$ direction for a short period $t_{z}\left(<<t_{m}\right)$ and then flipped back to the transverse plane for detection. If segmental reorientations occur during $t_{m}$, the orientation-dependent frequency changes and the CSA is not completely refocused. The resulting dephasing of the stimulated-echo is observed as a decrease in the detected spectral intensity. To remove $T_{1}$ and $T_{2}$ relaxation effects during $t_{m}$ and $\tau_{C S A}$, respectively, a reference spectrum $S_{0}=S\left(t_{z}, \delta \tau_{C S A}\right)$, which has the same relaxation factors, but no motion effects during $t_{m}$, is measured. $S_{0}$ is obtained by simply swapping $t_{m}$ and $t_{z}$. To remove the contribution of segments that do not move on the milliseconds to seconds time scale, the 1D PUREX intensity $S=S\left(t_{m}, \delta \tau_{C S A}\right)$ is subtracted from the reference spectrum $S_{0}$. Dividing this difference by $S_{0}$, one can obtain a normalized pure-exchange intensity $E\left(t_{m}, \delta \tau_{C S A}\right)=\left(S_{0}-S\right) / S_{0}$, which does not contain relaxation effects and just accounts for the signals from the reorienting segments. For discrete jumps with correlation times $\tau_{c}$ in the range of milliseconds to seconds (slow-motion regime), the normalized pure-exchange intensity $E\left(t_{m}, \delta \tau_{C S A}\right)$ can be approximated by the following expression:

$$
E\left(t_{m}, \delta \tau_{C S A}\right)=
$$

$$
=\frac{f_{m}(M-1)}{M}\left\{1-\Phi\left(t_{m}\right)\right\}\left\langle 2 \sin ^{2}\left[\left(\omega_{1}-\omega_{2}\right) \frac{\tau_{C S A}}{4}\right]\right\rangle
$$

where $f_{m}$ is the fraction of slow mobile segments, (mobile fraction), $M$ is the number of magnetically non equivalent sites accessible to the moving segments, $\Phi\left(t_{m}\right)$ is the correlation function of the molecular motion, and $\left(\omega_{1}-\omega_{2}\right)$ is the difference between the NMR frequencies before and after $t_{m}$. Therefore, the $t_{m}$ dependence of $E\left(t_{m}, \delta \tau_{C S A}\right)$ yields the time scale of the motion, while its $\tau_{C S A}$ dependence provides information on the magnitude of $\left(\omega_{1}-\omega_{2}\right)$, which is proportional to the amplitude of the slow molecular reorientations that occur during $t_{m}$.

An example of the application of 1D PUREX NMR in the study of a discrete jump process is the case of the molecular motions responsible by the $\beta$-relaxation of poly(methyl methacrylate) (PMMA), see inset in Fig. 6b. PMMA belongs to an important class of glassy polymers, where mechanical and dielectric properties are strongly affected by the $\beta$-relaxation process, which is one of the most widely studied local relaxations in glassy polymers [41-43]. Because the exchange NMR experiments essentially detect reorientations of the ${ }^{13} \mathrm{C}$ chemical shift anisotropy (CSA) tensors, the molecular motion occurring as part of the $\beta$-relaxation can be well probed by the carbonyl groups $\mathrm{COO}$.

Figure $6 \mathrm{~b}$ (left) shows the reference, $S_{0}$, the 1D PUREX, $S$, and the difference, $S_{0}-S,{ }^{13} \mathrm{C}$ spectra obtained for PMMA at $\mathrm{T}=295 \mathrm{~K}$. The $S_{0}-S$ spectrum shows significant intensity for all carbons confirming that the $\beta$-relaxation of PMMA involves both the side group and main chain motions. The $\tau_{C S A}$-dependence of $E\left(t_{m}, \delta \tau_{C S A}\right)$ for the carbonyl group in PMMA is shown in Fig. 6b (right). The bimodal character of the curve is promptly recognized, showing the presence of both small (slow build-up) and high (fast build-up) angular motions. The $\beta$-relaxation of PMMA involves rotations of the ester side group around the $\mathrm{C}-\mathrm{COO}$ bond ( $\pi$-flip) accompanied by rearrangements of the main chain (small-angle twists), which occur in several steps: starting from a given orientation, 
the asymmetric ester side group executes a flip coupled to a twist around the main chain axis (component I), which avoids a steric clash with the local environment. Subsequently, after a new flip (flip-back), also accompanied by small-angle twist, the side group orientation is slightly different from its original position (component II), which results in a small angle reorientation of the carbonyl group. Another fraction of the molecular segments do not executes flips, but only twists around the main chain (component III). The best-fit simulation of the $E\left(t_{m}, \delta \tau_{C S A}\right)$ 1D PUREX intensities as a function of $\tau_{C S A}$ using this model is also shown in Fig. 6b $[42,43]$. All small-angle rotations were simulated using Gaussian distributions of reorientation angles centered at $0^{\circ}$ with root-meansquare widths $\sigma$. The $E\left(t_{m}, \delta \tau_{C S A}\right)$ vs. $\tau_{C S A}$ simulated curves for each component are also shown in Fig. $6 \mathrm{~b}$. These results are in very good agreement with those ones obtained by 2D Exchange NMR in ${ }^{13} \mathrm{C}$ labeled samples [41].

Although being a more efficient method for detecting and characterizing small moving fractions, the problem concerning the low site resolution still remains in the 1D PUREX experiment. This is particularly problematic for ${ }^{13} \mathrm{C}$ studies of chemically complex systems, where the overlap between the powder patterns of different chemical groups migth make impossible to study motions occurring at different sites independently. This problem was overcome by a class of methods called Centerband-Only Detection of Exchange (CODEX), where slow molecular motions are quantified based on the intensities of the isotropic lines (centerband) in a high resolution MAS spectrum, without the necessity of observing MAS sidebands $[35,36,44]$. The CODEX experiment is essentially the MAS version of 1D PUREX NMR. However, the averaging of the chemical-shift anisotropy under MAS provides higher site resolution and sensitivity. On the other hand, MAS makes the NMR frequency insensitive to segmental orientations and reorientations. Thus, it is necessary to reintroduce (recouple) the chemical shift anisotropy during the periods used to codify the exchange process, i.e., the evolution periods. This is performed by using a train of rotor synchronized $180^{\circ}$ pulses capable of reintroducing the chemical shift anisotropy even under high MAS spinning frequencies, Fig. 6c. This scheme, known as recoupling of the CSA, was originally developed for using in the so-called Rotational-Echo Double-Resonance (REDOR) experiments, which are a very useful class of NMR methods for measuring internuclear distances [45]. The CSA recoupling during the evolution periods is achieved by replacing each evolution period $\tau$ in the 1D PUREX pulse sequence by a train of $N / 2180^{\circ}$ pulses spaced by $t_{r} / 2$, where $t_{r}$ is the sample rotation period, Fig. 6c. This leads to a total evolutionand-refocusing period of $N t_{r}$. With the CSA recoupling, the phase accumulated during the first and second evolution periods before, $\Phi_{1}$, and after, $\Phi_{2}$, the mixing time are given by:

$$
\begin{aligned}
\Phi_{1} & =\frac{N}{2}\left\{-\int_{0}^{t_{r} / 2}\left[\omega_{\text {iso }}+\omega_{\text {aniso }, 1}(t)\right] d t+\int_{t_{r} / 2}^{t_{r}}\left[\omega_{\text {iso }}+\omega_{\text {aniso }, 1}(t)\right] d t\right\}= \\
& =-N \int_{0}^{t_{r} / 2} \omega_{\text {aniso }, 1}(t) d t \\
\Phi_{2} & =\frac{N}{2}\left\{-\int_{0}^{t_{r} / 2}\left[\omega_{\text {iso }}+\omega_{\text {aniso }, 2}(t)\right] d t+\int_{t_{r} / 2}^{t_{r}}\left[\omega_{\text {iso }}+\omega_{\text {aniso }, 2}(t)\right] d t\right\}= \\
& =-N \int_{0}^{t_{r} / 2} \omega_{\text {aniso }, 2}(t) d t
\end{aligned}
$$

where $\omega_{\text {aniso, } 1}(t)$ and $\omega_{\text {aniso,2 }}(t)$ are the NMR frequencies due to the anisotropic part of the chemical shift under MAS, before and after $t_{m}$, respectively.

$$
\omega_{\text {aniso }, i}(t)=\omega_{0}\left\{\sum_{k=1}^{2} C_{k}^{i} \cos \left[k\left(\omega_{r} t+\gamma\right)\right]+C_{k}^{i} \sin \left[k\left(\omega_{r} t+\gamma\right)\right]\right\}
$$

The elements $C_{k}$ and $S_{k}$ depend on the elements of the anisotropic interaction tensor, expressed in the molecular frames of the exchanging sites, as well as on the Euler angles $\alpha$ and $\beta$. Together with $\gamma$, these specify the orientation of a given molecular frame with respect to the rotor frame [2]. The periodicity of the MAS signal with the MAS-rotation periods 


$$
\Phi=\int_{0}^{t_{r}} \omega(t) d t=0
$$

was used to derive the expressions for the phases. If $\Phi_{1}=\Phi_{2}$, i.e., if no exchange occurs the total phase accumulation is $\Phi_{1}+\Phi_{2}=\left|\Phi_{1}\right|-\left|\Phi_{2}\right|=0$; in other words, the magnetization is refocused in a stimulated-echo along its original direction. Molecular motions during $t_{m}$ reduce the stimulatedecho and the observed line intensities. Therefore, the analysis of the motional amplitude and time scale can be performed in the same way as for the 1D PUREX measurements. The CODEX intensity $S=S\left(t_{m}, \delta N t_{r}\right)$ and the reference intensity with no motion effects, $S_{0}=S\left(t_{z}, \delta N t_{r}\right)$, also obtained after swapping $t_{m}$ and $t_{z}$, are combined to produce the normalized pure exchange intensity, $E\left(t_{m}, \delta N t_{r}\right)=\left(S_{0}-S\right) / S_{0}$. A plot of $E\left(t_{m}, \delta N t_{r}\right)$ as a function of $t_{m}$ or $N t_{r}$ provides information on the motional rate and amplitude, respectively. The main advantage of CODEX is the possibility of studying the molecular motion of each chemical site independently, since a high resolution MAS spectrum is acquired. This makes it possible to probe local motions at each individual site of chemically complex systems. A CODEX spectrum is similar to a regular $\mathrm{CP} / \mathrm{MAS}$ spectrum with reduced intensities due to the dephasing by molecular motion.

Figures $6 \mathrm{~d}$ shows the experimental and simulated $E\left(t_{m}, \delta N t_{r}\right)$ vs. $N t_{r}$ CODEX curves for protonated (2, 3, 5, and 6 ) and non-protonated (1 and 4) phenylene carbons in the PPV polymer [31]. It is not possible to fit the experimental data considering only ring flips $\left(180^{\circ}\right.$ rotation around the 1,4 axis), indicating that there are some segments also executing smallangle motions. Thus, in Fig. 6d the long-dashed line correspond to groups executing only flips, while the short-dashed line accounts for groups just executing small-angle motions. From the fitting of both protonated and non-protonated carbons CODEX curves the average amplitude of the flip imprecision was estimated as $\bar{\psi}_{i m p}=(30 \pm 5)^{\circ}$. The fraction of segments executing small-angle oscillations was evaluated to be $f_{m}^{o s c}=(0.3 \pm 0.1)$, and the average amplitude of these oscillations was found to be $\bar{\psi}_{o s c}=(9 \pm 2)^{\circ}$.

Many extensions of the CODEX technique have appeared, including a four-time pulse sequence used for studying motional memory [36], a triple resonance CODEX applied to study protein dynamics [46,47], a scaled CODEX efficiently used at low spinning rates [38], a CODEX used for torsion angles determination in solids [48], a ${ }^{19} \mathrm{~F}$ CODEX used to study the oligomerization in lipid bilayers proteins [49], and a CODEX variant to measure internuclear distances between a spin $1 / 2$ and a quadrupolar nuclei [50].

\section{CONFORMATION}

The experimental elucidation of molecular conformations in unoriented solids, such as amorphous polymers or polycrystalline proteins, is a challenge. Solid-state NMR provides a)

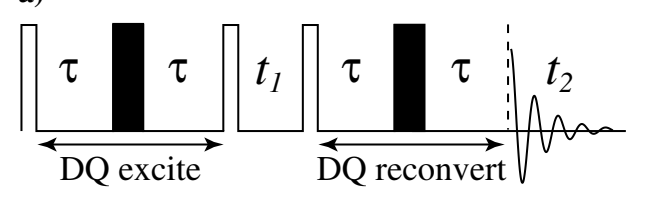

b)
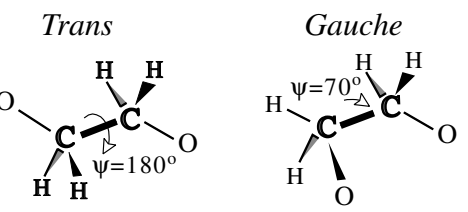

FIG. 7: a) INADEQUATE pulse sequence. Black and white pulses represent $180^{\circ}$ and $90^{\circ}$ pulses, respectively; b) Trans and gauche conformations in PEO.

a set of techniques that show great potential for determining molecular conformations on the level of individual bonds, by probing relative segmental orientations or internuclear distances through various anisotropic nuclear interactions, such as dipolar couplings and chemical shifts. Particularly, detailed information on torsion angles and their distributions can be obtained by correlating NMR interaction-tensor orientations in two-dimensional NMR spectra without magic-angle spinning. The techniques are based on 2D Exchange NMR $[2,23,25-28]$ or on correlation of dipolar couplings with the chemical shifts $[51,52]$, mostly on specifically ${ }^{13} \mathrm{C}$ labeled samples.

Recently, one useful experiment to determine the torsion angle for ${ }^{13} \mathrm{C}-{ }^{13} \mathrm{C}$ pairs was proposed $[53,54]$ being very practical for providing information about the conformation of polymers [55-58]. This experiment, called Double-Quantum NMR (DQ-NMR) is based on the INADEQUATE $[59,60]$ pulse sequence (Fig. 7a). In order to perform the DQNMR experiment, the sample has to contain a pair of directly bonded ${ }^{13} \mathrm{C}$ nuclei with individual anisotropic chemical shifts $\omega_{a}$ and $\omega_{b}$, and coupled through dipolar interaction. The pulse sequence consists of two identical blocks, which include two evolution times $\tau$ separated by a $\pi$ pulse $(\tau-\pi-\tau)$. The first $(\tau-\pi-\tau)$ block produces a non-observable doublequantum coherence, represented in this case by the sum of the anisotropic chemical shifts of the dipolar coupled sites $\left(\omega_{a}+\omega_{b}\right)$, which evolves during the period $t_{1}$. Subsequently, the double-quantum coherence is reconverted, through the second $(\tau-\pi-\tau)$ block, into an observable magnetization, which is modulated by the double-quantum coherence and measured during the detection period $t_{2}$. The signal acquired during $t_{2}, f\left(t_{2}\right)$, under continuous-wave proton decoupling, involves the individual anisotropic chemical shifts $\omega_{a}$ and $\omega_{b}$ , and the ${ }^{13} \mathrm{C}-{ }^{13} \mathrm{C}$ dipole-dipole interaction. In an experiment with complete dipolar decoupling during detection [54], the signal involves only the individual anisotropic chemical shifts $\omega_{a}$ and $\omega_{b}, f\left(t_{2}\right)=\exp \left(i \omega_{a} t_{2}\right)+\exp \left(i \omega_{b} t_{2}\right)$, so that in the 2D spectrum the intensities are observed at $\left(\omega_{a}+\omega_{b}, \omega_{a}\right)$ and $\left(\omega_{a}+\omega_{b}, \omega_{b}\right)$. Therefore, the pulse sequence yields 2D spectra where the first spectral dimension, $\omega_{1}$, reflects the torsion angle in terms of the sum of the anisotropic chemical shifts of 


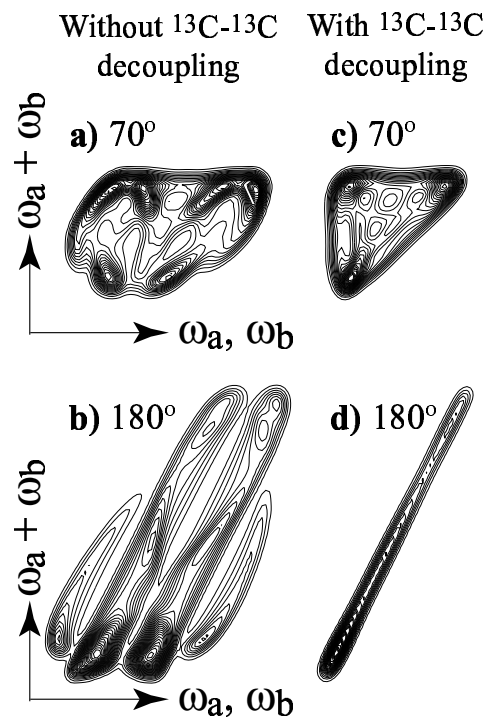

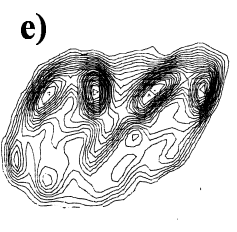

f)

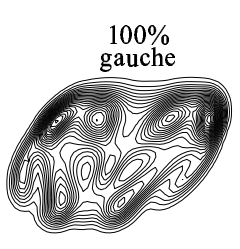

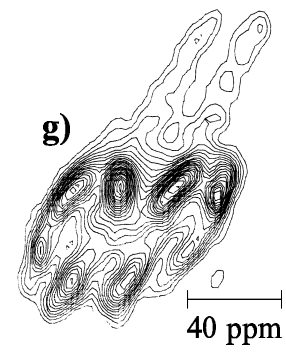

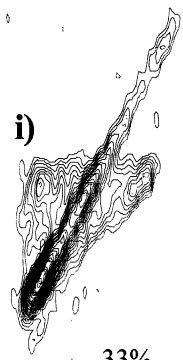

h)

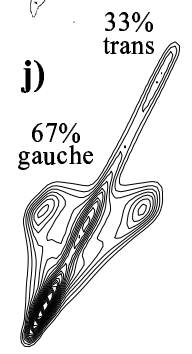

FIG. 8: Simulated ${ }^{13} \mathrm{C}$ DQ-NMR spectra of PEO with and without ${ }^{13} \mathrm{C}_{-}{ }^{13} \mathrm{C}$ dipolar decoupling for torsion angles of (a/c) $70^{\circ}$ (gauche) and (b/d) $180^{\circ}$ (trans). e) ${ }^{13} \mathrm{C}$ DQ-NMR spectrum of PEO/RES without decoupling measured at $75.5 \mathrm{MHz}$ and $250 \mathrm{~K}$. f) The best simulation for spectrum shown in e). ${ }^{13} \mathrm{C}$ DQ-NMR spectra of PEO/PNP measured at $75.5 \mathrm{MHz}$ and $293 \mathrm{~K}$ : g) without decoupling and i) with decoupling. The best simulations of both the undecoupled (h) and decoupled (j) spectra.(Adapted with permission from reference [56] - Copyright 2005 American Chemical Society )

the dipolar coupled sites $\left(\omega_{a}+\omega_{b}\right)$, without any interference from the dipolar coupling. The conformational information is enhanced by the correlation with the individual anisotropic chemical shifts in the directly detected $\omega_{2}$ dimension $\left(\omega_{a}\right.$ and $\omega_{b}$ ), with or without ${ }^{13} \mathrm{C}-{ }^{13} \mathrm{C}$ homonuclear decoupling [54]. The application of this experiment is going to be demonstrated on polyethylene oxide (PEO) with dilute $(\sim 13 \%){ }^{13} \mathrm{C}-{ }^{13} \mathrm{C}$ spin pairs in different chemical environments in the crystalline and amorphous phase, determining the amount of trans (T) and gauche $(\mathrm{G})$ conformations (Fig. 7b).

PEO chains in the crystalline state have been studied by infrared, Raman, and X-ray techniques [61,62]. The results have shown that the chains adopt a $7_{2}$ helix conformation with a TTG sequence: all OC-CO bonds are gauche and all $\mathrm{CO}-\mathrm{CC}$ bonds are trans. However, the helix is distorted to improve intermolecular interactions. A detailed analysis of the $\mathrm{X}$-ray diffraction data suggested a possible model for chain conformation: the helix is highly distorted with the OC-CO bonds having discrete torsion angles of $57.0^{\circ}, 67.8^{\circ}, 74.2^{\circ}$, $49.0^{\circ}, 91.8^{\circ}, 60.2^{\circ}$, and $79.1^{\circ}[63]$. 2D DQ-NMR spectra indicated that the OC-CO bonds are all gauche with an average torsion angle of $\Psi=(74 \pm 4)^{\circ}$ and a narrow torsion angle distribution, $\sigma_{\Psi}<8^{\circ}$ [58]. This is different from the wider range of gauche torsion angles in the distorted helical structure previously proposed based on X-ray fiber diffraction [63]. Other interesting materials to be studied by DQ-NMR are the crystalline complexes of PEO with resorcinol (RES) and $p$-nitrophenol (PNP). WAXD and IR data suggest that the conformation of the PEO in the complex with RES is only slightly distorted from the original 72 helix [64]. The conformation of the chains in these systems remains TTG but the periodicity of the helix changes slightly [65]. Contrary to the PEO/RES complex, the conformation of the chains in $\mathrm{PEO} / \mathrm{PNP}$ complexes is very different from the $7_{2}$ helical conformation [66]. IR and WAXD analyses suggested that $1 / 3$ of the OC-CO bonds are in the trans conformation. The hydrogen bonding between the host and guest molecules is believed to induce the chain to adopt a TTGTTGTTT TTG'TTG'TTT structure [67].

Examples of simulated DQ-NMR spectra obtained for gauche and trans torsion angles of $70^{\circ}$ and $180^{\circ}$, respectively, for the $\mathrm{O}^{13} \mathrm{C}-{ }^{13} \mathrm{CO}$ bond are shown in Figs. 8a through d [56]. The observed and simulated ${ }^{13} \mathrm{C}-{ }^{13} \mathrm{C}$ undecoupled DQ-NMR spectra of PEO/RES are shown in Figs. 8e and f. The observed spectrum shows that all of the OC-CO torsion angles are gauche. The simulation suggests that the average torsion angle is $(\Psi=74 \pm 9)^{\circ}$ with an angle distribution width of $\sigma<10^{\circ}$. The observed and simulated undecoupled and decoupled static DQ-NMR spectra of PEO/PNP are shown in Figs. $8 \mathrm{~g}$ through $\mathrm{j}$. The spectra show a strong slope-two diagonal ridge due to trans OC-CO bonds. The simulations of the observed spectra were produced by factoring 0.33 for trans and 0.67 for gauche conformers. Both the undecoupled and decoupled static DQ-NMR spectra are consistent with one third of OC-CO bonds of the PEO/PNP in the trans conformation $\left(\Psi=180^{\circ}, \sigma<7^{\circ}\right)$, and two-thirds in the gauche conformation $\left(\Psi=70 \pm 9^{\circ}, \sigma<10^{\circ}\right)$. The $2 \mathrm{D}^{13} \mathrm{C}$ DQ-NMR spectrum confirmed that the OC-CO torsion angles in PEO/RES are all gauche. In contrast, one third of the OC-CO torsion angles in PEO/PNP are trans and two thirds are gauche, confirming the previously suggested structure [67]. ${ }^{13} \mathrm{C}$ DQ-NMR experiments were additionally performed for studying amorphous PEO intercalated in clays and $\mathrm{MoS}_{2}$ and the spectra indicated that in both situations approximately $10 \%$ the OC-CO tor- 
sion angles are in the trans conformation [55]. Moreover, this method was used successfully to determine the conformation of other polymers $[48,68,69]$ and biomaterials [70,71].

\section{CONCLUSIONS AND OUTLOOK}

In this article basic and advanced aspects of modern multidimensional multiple-resonance NMR methodologies, used to the study molecular dynamics and conformation in solid materials, have been described. The use of such methods for studying solid polymers and derivatives has been illustrated by several examples. A remarkable characteristic of new NMR methods is the possibility of getting important information about molecular structure and dynamics of materials in an al- most model free fashion, making NMR a unique tool for the characterization of complex materials. Besides, despite its 50 years of history, the development of new NMR methods is still and opened area, being one of the main challenges for NMR researchers.

\section{ACKNOWLEDGMENTS}

The authors would like to dedicate this article to the memory of Professor Horácio C. Panepucci, who played the major role in several periods of their professional careers. The authors acknowledge support from CAPES, CNPq, and FAPESP.
[1] U. Haeberlen, High Resolution NMR in Solids, Advances in Magnetic Resonance, Academic Press, New York, 1st edition, (1976).

[2] K. Schmidt-Rohr, and H. W. Spiess, Multidimensional SolidState NMR and Polymers, Academic Press, London, 1st edition, (1994).

[3] E. R. deAzevedo, T. J. Bonagamba, and D. Reichert, Prog. Nucl. Magn. Reson. Spectrosc., 47, 137 (2005).

[4] G. Lipari, A. Szabo, and R. M. Levy, Nature, 300, 197 (1982).

[5] C. P. Slichter, Principles of Magnetic Resonance, volume 1, Springer Series in Solid-State Sciences, Springer-Verlag, Berlin, 3rd edition, (1990).

[6] J. Schaefer, E. O. Stejskal, R. A. McKay, and W. T. Dixon, Journal of Magnetic Resonance, 57, 85 (1984).

[7] M. Hong, X. L. Yao, K. Jakes, and D. Huster, J. Phys. Chem. B, 106, 7355 (2002)

[8] A. Abragam, Principles of Nuclear Magnetism, volume 32, International Series of Monographs, Oxford University Press, Oxford, edition, (1994)

[9] E. R. deAzevedo, D. Reichert, E. L. G. Vidoto, K. Dahmouche, P. Judeinstein, and T. J. Bonagamba, Chem. Mater., 15, 2070 (2003).

[10] K. Dahmouche, M. Atik, N. C. Mello, T. J. Bonagamba, H. Panepucci, M. A. Aegerter, and P. Judeinstein, J. Sol.-Gel Sci.Technol., 8, 711 (1997).

[11] K. Dahmouche, M. Atik, N. C. Mello, T. J. Bonagamba, H. Panepucci, P. Judeinstein, and M. A. Aegerter, Sol. Energy Mater. Sol. Cells, 54, 1 (1998).

[12] N. C. Mello, T. J. Bonagamba, H. Panepucci, K. Dahmouche, P. Judeinstein, and M. A. Aegerter, Macromolecules, 33, 1280 (2000).

[13] P. H. de Souza, R. F. Bianchi, K. Dahmouche, P. Judeinstein, R. M. Faria, and T. J. Bonagamba, Chem. Mater., 13, 3685 (2001).

[14] M. G. Munowitz, R. G. Griffin, G. Bodenhausen, and T. H. Huang, J. Am. Chem. Soc., 103, 2529 (1981).

[15] J. Clauss, K. Schmidt-Rohr, and H. W. Spiess, Acta Polym., 44, 1 (1993).

[16] H. Kobayashi, S. Kanbe, S. Seki, H. Kigchi, M. Kimura, I. Yudasaka, S. Miyashita, T. Shimoda, C. R. Towns, J. H. Burroughes, and R. H. Friend, Synth. Met., 111, 125 (2000).

[17] A. R. Inigo, H.-C. Chiu, W. Fann, Y.-S. Huang, U.-S. Jeng, L. T-L, C.-H. Hsu, K.-Y. Peng, and S.-A. Chen, Phys. Rev. B, 69 , 075201 (2004).
[18] D. Huster, L. S. Xiao, and M. Hong, Biochemistry, 40, 7662 (2001).

[19] A. Bielecki, A. C. Kolbert, and M. H. Levitt, Chem. Phys. Lett., 155, 341 (1989).

[20] R. F. Cossiello, E. Kowalski, P. C. Rodrigues, L. Akcelrud, A. C. Bloise, E. R. deAzevedo, T. J. Bonagamba, and T. Atvars, Macromolecules, 38, 925 (2005)

[21] A. C. Bloise, E. R. deAzevedo, R. F. Cossiello, R. F. Bianchi, D. T. Balogh, R. M. Faria, T. D. Z. Atvars, and T. J. Bonagamba, Phys. Rev. B, 71, 174202 (2005).

[22] A. K. Sheridan, J. M. Lupton, I. D. W. Samuel, and D. D. C. Bradley, Chem. Phys. Lett., 322, 51 (2000).

[23] S. Kaufmann, S. Wefing, D. Schaefer, and H. W. Spiess, J. Chem. Phys., 93, 197 (1990).

[24] U. Tracht, H. Wilhelm, A. Heuer, and H. W. Spiess, J. Magn. Reson., 140, 460 (1999).

[25] Z. Luz, H. W. Spiess, and J. J. Titman, Isr. J. Chem., 32, 145 (1992).

[26] Z. Luz, P. Tekely, and D. Reichert, Prog. Nucl. Magn. Reson. Spectrosc., 41, 83 (2002).

[27] C. Schmidt, B. Blumich, and H. W. Spiess, J. Magn. Reson., 79, 269 (1988).

[28] H. W. Spiess, Ber. Bunsen-Ges. Phys. Chem., 101, 153 (1997).

[29] E. R. deAzevedo, T. J. Bonagamba, and K. Schmidt-Rohr, J. Magn. Reson., 142, 86 (2000).

[30] A. F. Dejong, A. P. M. Kentgens, and W. S. Veeman, Chem. Phys. Lett., 109, 337 (1984).

[31] E. R. deAzevedo, R. W. A. Franco, A. Marletta, R. M. Faria, and T. J. Bonagamba, J. Chem. Phys., 119, 2923 (2003).

[32] K. Zemke, K. Schmidt-Rohr, and H. W. Spiess, Acta Polym., 45, 148 (1994)

[33] Z. Olender, D. Reichert, A. Mueller, H. Zimmermann, R. Poupko, and Z. Luz, J. Magn. Reson., Ser. A, 120, 31 (1996).

[34] R. Bohmer, T. Jorg, F. Qi, and A. Titze, Chem. Phys. Lett., 316 419 (2000)

[35] E. R. deAzevedo, W. G. Hu, T. J. Bonagamba, and K. SchmidtRohr, J. Am. Chem. Soc., 121, 8411 (1999).

[36] E. R. deAzevedo, W. G. Hu, T. J. Bonagamba, and K. SchmidtRohr, J. Chem. Phys., 112, 8988 (2000).

[37] F. Fujara, S. Wefing, and H. W. Spiess, J. Chem. Phys., 84, 4579 (1986).

[38] D. Reichert, O. Pascui, T. J. Bonagamba, E. R. deAzevedo, and A. Schmidt, Chem. Phys. Lett., 380, 583 (2003). 
[39] D. Reichert, H. Zimmermann, P. Tekely, R. Poupko, and Z. Luz, J. Magn. Reson., 125, 245 (1997).

[40] E. R. deAzevedo, J. R. Tozoni, K. Schmidt-Rohr, and T. Bonagamba, J. Chem. Phys., 122, 154506 (2005).

[41] A. S. Kulik, H. W. Beckham, K. Schmidt-Rohr, D. Radloff, U. Pawelzik, C. Boeffel, and H. W. Spiess, Macromolecules, 27, 4746 (1994).

[42] T. J. Bonagamba, F. Becker-Guedes, E. R. DeAzevedo, and K. Schmidt-Rohr, J. Polym. Sci., Part B: Polym. Phys., 39, 2444 (2001).

[43] F. Becker-Guedes, E. R. deAzevedo, T. J. Bonagamba, and K. Schmidt-Rohr, Appl. Magn. Reson., 27, 383 (2004).

[44] K. Schmidt-Rohr, E. R. deAzevedo, and T. J. Bonagamba, Centerband-Only Detection of Exchange (CODEX): Efficient NMR Analysis of Slow Motions in Solids, In Encyclopedia of Nuclear Magnetic Resonance, volume 9: Advances in NMR, John Wiley and Sons, 633 (2002).

[45] T. Gullion, and J. Schaefer, J. Magn. Reson., 81, 196 (1989).

[46] E. R. deAzevedo, S. B. Kennedy, and M. Hong, Chem. Phys. Lett., 321, 43 (2000).

[47] A. P. M. Kentgens, E. deBoer, and W. S. Veeman, J. Chem. Phys., 87, 6859 (1987).

[48] H. Kaji, and K. Schmidt-Rohr, Macromolecules, 35, 7993 (2002).

[49] J. J. Buffy, A. J. Waring, and M. Hong, J. Am. Chem. Soc., 127, 4477 (2005).

[50] K. Saalwchter, and K. Schmidt-Rohr, J. Magn. Reson., 145, 161 (2000).

[51] D. P. Weliky, G. Dabbagh, and R. Tycko, J. Magn. Reson., Ser. A, 104, 10 (1993).

[52] T. Nakai, and C. A. MacDowell, Chem. Phys. Lett., 217, 234 (1994).

[53] K. Schmidt-Rohr, Macromolecules, 29, 3975 (1996).

[54] K. Schmidt-Rohr, J. Magn. Reson., 131, 209 (1998).
[55] D. J. Harris, T. J. Bonagamba, and K. Schmidt-Rohr, Macromolecules, 32, 6718 (1999).

[56] D. J. Harris, T. J. Bonagamba, M. Hong, and K. Schmidt-Rohr, Macromolecules, 33, 3375 (2000).

[57] D. J. Harris, T. J. Bonagamba, and K. Schmidt-Rohr, Macromolecules, 35, 5724 (2002).

[58] D. J. Harris, T. J. Bonagamba, M. Hong, and K. Schmidt-Rohr, Polymer, 46, 11737 (2005).

[59] A. Bax, R. Freeman, and S. P. Kempsell, J. Am. Chem. Soc., 102, 4849 (1980).

[60] T. Nakai, and C. A. McDowell, Mol. Phys., 79, 965 (1993).

[61] H. Tadokoro, Y. Chatani, M. Kobayashi, T. Yoshihara, S. Murahashi, and K. Amada, Rep. Prog. Polym. Phys. Jpn, 6, 303 (1963).

[62] H. Tadokoro, Y. Chatani, T. Yoshihara, S. Tahara, and S. Murahashi, Makromol. Chem., 74, 109 (1964).

[63] Y. Takahashi, and H. Tadokoro, Macromolecules, 6, 672 (1973).

[64] J. Spevacek, L. Paternostre, P. Damman, A. C. Draye, and M. Dosiere, Macromolecules, 31, 3612 (1998).

[65] R. M. Myasnikova, E. F. Titova, and E. S. Obolonkova, Polymer, 21, 403 (1980).

[66] J. J. Point, and C. Coutelier, J. Polym. Sci., Part B: Polym. Phys., 23, 231 (1985).

[67] P. Damman, and J. J. Point, Macromolecules, 26, 1722 (1993).

[68] M. G. Dunbar, B. M. Novak, and K. Schmidt-Rohr, Solid-State Nucl. Magn. Reson., 12, 119 (1998).

[69] H. Kaji, and K. Schmidt-Rohr, Macromolecules, 33, 5169 (2000).

[70] J. D. van Beek, L. Beaulieu, H. Schafer, M. Demura, T. Asakura, and B. H. Meier, Nature, 405, 1077 (2000).

[71] J. D. van Beek, B. H. Meier, and H. Schafer, J. Magn. Reson., 162, 141 (2003). 